\title{
Force/Vision Based Active Damping Control of Contact Transition in Dynamic Environments
}

\author{
Tomas Olsson, Rolf Johansson, Anders Robertsson \\ Department of Automatic Control, Lund Inst. of Tech., Lund University, SE-221 00 Lund, Sweden \\ E-mail: \{tomas.olsson, anders.robertsson, rolf.johansson\}@control.lth.se
}

\begin{abstract}
When a manipulator interacts with objects with poorly damped oscillatory modes, undesired oscillations and bouncing may result. In this paper, we present a method for observer-based control of a rigid manipulator interacting with an environment with linear dynamics. The controller injects a desired damping into the environment dynamics, using both visual- and force sensing for stable control of the contact transition. Stability of the system is shown using an observer-based backstepping design method, and simulations are performed in order to validate the chosen approach.
\end{abstract}

\section{INTRODUCTION AND PROBLEM FORMULATION}

Contact force control has long been an important research topic in robotics, and a large number of experimental and industrial implementations and applications have been presented in the literature [1]. Most of these methods focus on the robot dynamics, assuming that the environment can be modeled by ideal constraints, or as simple massless (linear or non-linear) spring-damper systems [2]. Dynamical systems can also be used to model many types of environments and the energy transfer between the robot and its environment [3], [4]. For efficient control of such dynamic environments it is necessary to consider not only the robot dynamics but also the dynamic properties of the environment itself, or otherwise undesirable phenomena such as bouncing may occur. The combination of vision and force sensing is very powerful, in that it allows us to control interaction with objects whose locations are not initially known, so that impact can be predicted and controlled in such a way that large impact forces are avoided.

There exist applications where force control in dynamic environments is required. As an industrial example we have compliant "tool adapters", which are mounted between a rigid workpiece and the fixture and serve as both an extra mechanical compliance and as a force sensor. Cooperating robots is another example, where one robot may be programmed with a desired compliance, holding a workpiece on which some operation is to be performed. Other examples are interaction with non-rigid structures, and loads suspended from cranes. In non-industrial environments, there are examples such as manipulation of flying, falling and rolling objects.
In uncertain but static environments, the force/vision control problem is often solved by assigning each available degree of freedom to a specific task, for instance by specifying it as either position- or force controlled as in [5]. The interaction control problem in dynamic environments is in general more challenging. In [4], dynamic effects such as friction in rigid body- and elastic contact with passive environments were analyzed, however only the contact phase of the interaction was considered, thereby avoiding the need for non-contact sensing such as vision. For interaction with non-stationary objects, for instance, it may be necessary to introduce feed-forward from the measured target motion during the approach phase, as in [6] where force/vision control was used for an assembly operation involving a moving target. In the most general case of interacting systems, however, the manipulator and environment dynamics are coupled through the forces of interaction, and feedback from multiple sensor signals may affect the overall stability and performance of the system in a complex way. As a way to overcome this difficulty, in [7] the use of vision/impedance control was used for pegin-hole insertion experiments in a stationary environment. However, as the range of achievable impedances is always limited by force sensor noise and dynamic effects such as gear box- and force sensor elasticity, as well as the presence of inner position feedback loops, a purely impedancebased approach may not be sufficient for efficient control in non-stationary environments.

\section{A. Problem Formulation}

In this paper, we present a method for observer-based control of a system consisting of a rigid manipulator interacting with an environment with linear dynamics, and demonstrate how vision and force can be used together in order to influence and control the dynamics of the environment, by active control during both the approachand contact phases. An observer-based backstepping procedure is used to obtain a control law which makes the manipulator/environment system asymptotically stable. The method is validated in simulations on a model of a simple three-link serial robot interacting with a massspring-damper system. 


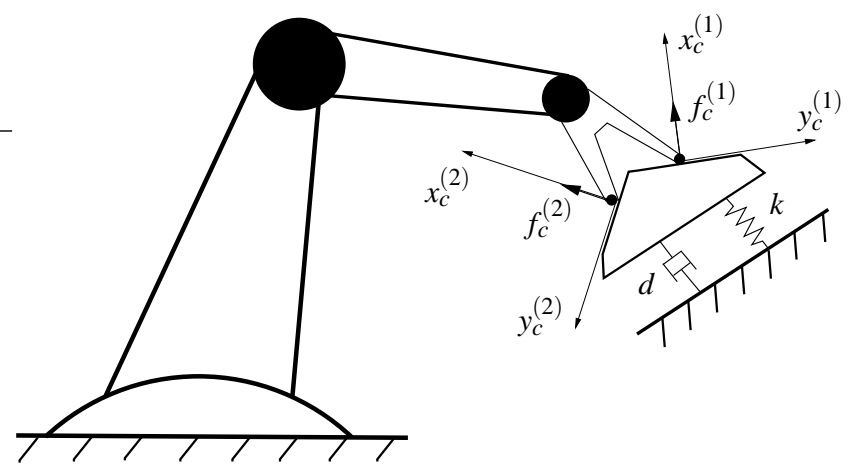

Fig. 1. Setup with multiple point contacts between robot and environment. Local coordinates $\left(x_{c}^{(i)}, y_{c}^{(i)}, z_{c}^{(i)}\right)$ are attached to the workpiece at each contact point, while the dynamics of the environment is modeled by a linear mass-spring-damper system .

\section{Modeling OF THE ROBOT AND ENVIRONMENT}

We assume the setup shown in Fig. 1. A rigid robot is in contact with a dynamic environment, where the interaction is modeled by contact forces in the surface normal direction at the contact points. The dynamics of a rigid robot can be modeled by the system

$$
\mathbf{M}(\mathbf{q}) \ddot{\mathbf{q}}+\mathbf{C}(\mathbf{q}, \dot{\mathbf{q}}) \dot{\mathbf{q}}+\mathbf{G}(\mathbf{q})=\tau-\mathbf{J}_{c}^{T}(\mathbf{q}) \mathbf{f}_{c}
$$

where $\mathbf{f}_{c}$ is the interaction (environment) force given in the chosen force space. By using a feedback linearizing control law it is possible to obtain a decoupled system of double integrators from a new command signal $\mathbf{u}$ to $\mathbf{x}_{c}$ [8]. Assuming robot double integrator dynamics with position $z_{3}$ and velocity $z_{4}$, and that the environment dynamics in one direction can be modeled as a stable linear second order system with position $z_{1}$ and velocity $z_{2}$, we can write the coupled dynamics of the robot and environment as

$$
\begin{aligned}
& \dot{z}_{1}=z_{2} \\
& \dot{z}_{2}=-k z_{1}-d z_{2}+\Psi\left(z_{3}-z_{1}\right) \\
& \dot{z}_{3}=z_{4} \\
& \dot{z}_{4}=u
\end{aligned}
$$

where the scalar contact force is

$$
f_{c}=\Psi(\delta) \stackrel{\text { def }}{=} K(\delta) \delta,
$$

where for convenience we have defined

$$
\delta \stackrel{\text { def }}{=} z_{3}-z_{1},
$$

and where the contact stiffness $K(\boldsymbol{\delta})$ is assumed to be a differentiable function of $\delta$, satisfying the properties

$$
\begin{aligned}
K(x) \geq 0, & & \forall x \\
K(x)=0, & & x<0 \\
|K(x)| \leq \bar{K}, & & \forall x \\
K^{\prime}(x) x+K(x)>0, & & x>0
\end{aligned}
$$

The final inequality expresses the condition that the contact force $f_{c}=K(\delta) \delta$ is increasing with respect to the deformation distance $\delta$. This also implies that the inverse $\Psi^{-1}\left(f_{c}\right)$ is well-defined when $\delta>0$. Eq. (6) together with properties (8)-(11) can be used as an approximate global model for a linear spring mechanism, or a local model for completely elastic non-linear contact of the type $f_{c}=k_{c} \delta_{c}^{n}$ with $n>1$ as described in [2].

Although the following section will use the simple model in Eq. (2)-(5), the method presented is straightforward to generalize to a larger class of systems, such as any passive lumped-multiple-mass environment model. The robot dynamics may in general be any linear secondorder system with relative degree two, which can always be transformed into a double integrator by a suitable state feedback. Such a model could for instance be used to model a position controlled manipulator with PD-control.

\section{CONTROLlER DESIGN}

\section{A. Full state feedback}

The aim of the controller is to obtain a sufficiently damped impact transition, which can be achieved by controlling the interaction forces suitably. The form of the system in Eq. (2)-(5) is similar to the so called strict feedback- or triangular form [9]. For such systems, the backstepping design method can be used to find a control law and a Lyapunov function in a recursive fashion. Due to the special structure of the passive environment, it makes sense to try to find a controller that aims to control the energy flow between the robot and environment such that the environment dynamics is sufficiently damped. If direct control of the interaction force $f_{c}$ was possible, we could introduce extra damping into the environment by choosing a "virtual" control signal of the form

$$
f_{c}=K\left(\alpha_{1}\left(z_{1}, z_{2}\right)-z_{1}\right) \cdot\left(\alpha_{1}\left(z_{1}, z_{2}\right)-z_{1}\right)
$$

with

$$
\alpha_{1}\left(z_{1}, z_{2}\right)=z_{1}+h\left(z_{2}\right)
$$

where the damping function $h\left(z_{2}\right)$ is twice continuously differentiable and chosen to satisfy the properties

$$
\begin{aligned}
h(x) & \geq 0, & & \forall x \\
h(x) & =0, & & x>0 \\
|d h(x) / d x| & <\bar{h}, & & \forall x
\end{aligned}
$$

In this way, extra damping is introduced by a suitable dissipation of energy by application of a contact force in the opposite direction of motion during the part of the motion when the contact point velocity $z_{2}<0$. This can be seen by introducing the energy-based Lyapunov function

$$
V_{1}\left(z_{1}, z_{2}\right)=\frac{1}{2} k z_{1}^{2}+\frac{1}{2} z_{2}^{2}
$$


which gives

$$
\begin{aligned}
\dot{V}_{1} & =k z_{1} z_{2}+z_{2}\left(-k z_{1}-d z_{2}+K(\boldsymbol{\delta})\left(\varepsilon_{3}+h\left(z_{2}\right)\right)\right. \\
& =\underbrace{-d z_{2}^{2}+z_{2} K(\delta) h\left(z_{2}\right)}_{\stackrel{\text { def }}{=}-W\left(z_{2}, \varepsilon_{3}\right) \leq 0}+z_{2} K(\delta) \varepsilon_{3}
\end{aligned}
$$

where the term $z_{2} K(\delta) h\left(z_{2}\right) \leq 0$ introduces extra damping due to properties (8), (14)-(15), and where

$$
\varepsilon_{3}=z_{3}-\alpha_{1}\left(z_{1}, z_{2}\right)=z_{3}-z_{1}-h\left(z_{2}\right),
$$

is interpreted as the error between the tool tip position corresponding to the "virtual" control signal and the true position. We can now write Eq. (3) as

$$
\dot{z}_{2}=-k z_{1}-d z_{2}+K(\delta) h\left(z_{2}\right)+K(\delta) \varepsilon_{3} \stackrel{\text { def }}{=} f_{2}\left(z_{1}, z_{2}, \varepsilon_{3}\right)
$$

Furthermore, we have

$$
\dot{\varepsilon}_{3}=z_{4}-\dot{\alpha}_{1}=z_{4}-z_{2}-h^{\prime}\left(z_{2}\right) f_{2}\left(z_{1}, z_{2}, \varepsilon_{3}\right)
$$

By augmenting $V_{1}$ with a quadratic term in $\varepsilon_{3}$, we obtain

$$
V_{2}\left(z_{1}, z_{2}, \varepsilon_{3}\right)=V_{1}\left(z_{1}, z_{2}\right)+\frac{1}{2} p_{3} \varepsilon_{3}^{2}, \quad p_{3}>0
$$

which gives

$$
\begin{aligned}
\dot{V}_{2} & =-W\left(z_{2}, \varepsilon_{3}\right)+\varepsilon_{3} K(\boldsymbol{\delta}) z_{2}+p_{3} \varepsilon_{3}\left(z_{4}-\dot{\alpha}_{1}\right) \\
& =-W\left(z_{2}, \varepsilon_{3}\right)+p_{3} \varepsilon_{3}\left(p_{3}^{-1} K(\boldsymbol{\delta}) z_{2}+z_{4}-\dot{\alpha}_{1}\right) \\
& =-W\left(z_{2}, \varepsilon_{3}\right)-p_{3} k_{3} \varepsilon_{3}^{2}+p_{3} \varepsilon_{3} \varepsilon_{4}
\end{aligned}
$$

with $k_{3}>0$ and the new error signal $\varepsilon_{4}$ given by

$$
\varepsilon_{4}=z_{4}-\dot{\alpha}_{1}+k_{3} \varepsilon_{3}+p_{3}^{-1} K(\delta) z_{2} .
$$

Eqs. (21) and (24) give

$$
\dot{\varepsilon}_{3}=z_{4}-\dot{\alpha}_{1}=-k_{3} \varepsilon_{3}+\varepsilon_{4}-p_{3}^{-1} K(\delta) z_{2} \stackrel{\text { def }}{=} f_{3}(\mathbf{z}, \varepsilon) .
$$

Differentiating Eq. (24) gives

$$
\begin{aligned}
\dot{\varepsilon}_{4} & =u-\ddot{\alpha}_{1}+k_{3} f_{3}(\mathbf{z}, \varepsilon)+p_{3}^{-1}\left(K(\boldsymbol{\delta}) f_{2}\left(z_{1}, z_{2}, \varepsilon_{3}\right)+\right. \\
& \left.+K^{\prime}(\boldsymbol{\delta})\left(f_{3}(\mathbf{z}, \boldsymbol{\varepsilon})+h^{\prime}\left(z_{2}\right) f_{2}\left(z_{1}, z_{2}, \varepsilon_{3}\right)\right) z_{2}\right)
\end{aligned}
$$

with

$$
\begin{aligned}
& \ddot{\alpha}_{1}=f_{2}\left(\mathbf{z}, \varepsilon_{3}\right)+h^{\prime \prime}\left(z_{2}\right) f_{2}\left(\mathbf{z}, \varepsilon_{3}\right)^{2}+h^{\prime}\left(z_{2}\right)\left(-k z_{2}-\right. \\
& \left.\quad d f_{2}\left(\mathbf{z}, \varepsilon_{3}\right)+\left(K^{\prime}(\boldsymbol{\delta})\left(\varepsilon_{3}+h\left(z_{2}\right)\right)+K(\boldsymbol{\delta})\right) f_{3}(\mathbf{z}, \boldsymbol{\varepsilon})\right) .
\end{aligned}
$$

By augmenting $V_{2}$ with a quadratic term in the error $\varepsilon_{4}$

$$
V_{3}\left(z_{1}, z_{2}, \varepsilon_{3}, \varepsilon_{4}\right)=V_{2}\left(z_{1}, z_{2}, \varepsilon_{3}\right)+\frac{1}{2} p_{4} \varepsilon_{4}^{2}, \quad p_{4}>0
$$

we get

$$
\begin{gathered}
\dot{V}_{3}=\dot{V}_{2}+p_{4} \varepsilon_{4} \dot{\varepsilon}_{4}=-W\left(z_{2}, \varepsilon_{3}\right)-k_{3} p_{3} \varepsilon_{3}^{2}+p_{4} \varepsilon_{4} \times \\
\quad\left(p_{3} p_{4}^{-1} \varepsilon_{3}+u-\ddot{\alpha}_{1}-k_{3}^{2} \varepsilon_{3}+k_{3} \varepsilon_{4}-k_{3} p_{3}^{-1} K(\boldsymbol{\delta}) z_{2}+\right. \\
\left.p_{3}^{-1}\left(K(\boldsymbol{\delta}) f_{2}+K^{\prime}(\boldsymbol{\delta})\left(f_{3}(\mathbf{z}, \varepsilon)+h^{\prime}\left(z_{2}\right) f_{2}\right) z_{2}\right)\right)
\end{gathered}
$$

We can now choose the control signal

$$
\begin{aligned}
u & =-\left(k_{3}+k_{4}\right) \varepsilon_{4}+\ddot{\alpha}_{1}+k_{3}^{2} \varepsilon_{3}+k_{3} p_{3}^{-1} K(\delta) z_{2}-p_{3}^{-1} \times \\
& \times\left(K(\delta) f_{2}\left(\mathbf{z}, \varepsilon_{3}\right)+K^{\prime}(\delta)\left(-k_{3} \varepsilon_{3}+\varepsilon_{4}-p_{3}^{-1} K(\delta) z_{2}\right.\right. \\
& \left.\left.+h^{\prime}\left(z_{2}\right) f_{2}\left(z_{1}, z_{2}, \varepsilon_{3}\right)\right) z_{2}\right)
\end{aligned}
$$

with $k_{4}>0$ and obtain

$$
\dot{V}_{3}=-W\left(z_{2}, \varepsilon_{3}\right)-k_{3} p_{3} \varepsilon_{3}^{2}-k_{4} p_{4} \varepsilon_{4}^{2}+p_{3} \varepsilon_{3} \varepsilon_{4}
$$

which is negative semidefinite if the free parameter $p_{4}$ satisfies $p_{4} \geq p_{3} /\left(4 k_{3} k_{4}\right)$. Asymptotic stability of the origin $z_{1}=z_{2}=\varepsilon_{3}=\varepsilon_{4}=0$ follows from LaSalle's theorem [9], since the largest invariant set in $\left\{(z, \varepsilon) \mid \dot{V}_{3}=0\right\}$ is the origin.

\section{B. Observer-based design}

Extension can be made to the case when the environment state is not directly measurable, assuming that the contact force $f_{c}$ is measurable, and when a (potentially noisy) position measurement $z_{1}$ is available from the camera. In this case, a linear globally exponentially convergent full state observer for the environment can be constructed as

$$
\begin{aligned}
& \dot{\hat{z}}_{1}=\hat{z}_{2}+l_{1}\left(z_{1}-\hat{z}_{1}\right) \stackrel{\text { def }}{=} \bar{f}_{1}\left(z_{1}, \hat{\mathbf{z}}\right) \\
& \dot{\hat{z}}_{2}=-k \hat{z}_{1}-d \hat{z}_{2}+f_{c}+l_{2}\left(z_{1}-\hat{z}_{1}\right) \stackrel{\text { def }}{=} \bar{f}_{2}\left(z_{1}, \hat{\mathbf{z}}, f_{c}\right)
\end{aligned}
$$

which gives the error dynamics

$$
\begin{aligned}
& \dot{\tilde{z}}_{1}=\tilde{z}_{2}-l_{1} \tilde{z}_{1} \\
& \dot{\tilde{z}}_{2}=-k \tilde{z}_{1}-d \tilde{z}_{2}-l_{2} \tilde{z}_{1}
\end{aligned}
$$

which can be made to converge exponentially to zero by choosing the (possibly time-varying) observer gains $l_{1}$ and $l_{2}$ suitably. By defining

$$
\alpha_{1}\left(\hat{z}_{1}, \hat{z}_{2}\right)=\hat{z}_{1}+h\left(\hat{z}_{2}\right)
$$

and

$$
\varepsilon_{3}=z_{3}-\alpha_{1}\left(\hat{z}_{1}, \hat{z}_{2}\right)=z_{3}-\hat{z}_{1}-h\left(\hat{z}_{2}\right)
$$

we can write Eq. (3) as

$$
\begin{aligned}
\dot{z}_{2}= & -k z_{1}-d z_{2}+K(\delta)\left(\varepsilon_{3}+h\left(\hat{z}_{2}\right)-\tilde{z}_{1}\right) \\
= & -k z_{1}-d z_{2}+K(\delta) h\left(z_{2}\right)+K(\delta) \varepsilon_{3} \\
& +K(\delta)\left[h\left(\hat{z}_{2}\right)-h\left(z_{2}\right)\right]-K(\delta) \tilde{z}_{1}
\end{aligned}
$$

Using the Lyapunov function $V_{1}$ of Eq. (17) gives

$$
\begin{aligned}
\dot{V}_{1} & =-W\left(z_{2}, \varepsilon_{3}\right)+\underbrace{z_{2} K(\boldsymbol{\delta})\left[h\left(\hat{z}_{2}\right)-h\left(z_{2}\right)\right]-z_{2} K(\boldsymbol{\delta}) \tilde{z}_{1}}_{W_{z \tilde{z}}(\mathbf{z}, \tilde{\mathbf{z}})}+ \\
& +z_{2} K(\boldsymbol{\delta}) \varepsilon_{3},
\end{aligned}
$$

and using $V_{2}$ in Eq. (22) we find

$$
\begin{aligned}
\dot{V}_{2} & =-W+W_{z \tilde{z}}+p_{3} \varepsilon_{3}\left(p_{3}^{-1} K(\delta) z_{2}+z_{4}-\dot{\alpha}_{1}\right) \\
& \stackrel{\text { def }}{=}-W-p_{3} k_{3} \varepsilon_{3}^{2}+p_{3} \varepsilon_{3} \varepsilon_{4}+W_{z}+\varepsilon_{3} K(\delta) \tilde{z}_{2}
\end{aligned}
$$


where we have defined

$$
\varepsilon_{4}=z_{4}-\dot{\alpha}_{1}+k_{3} \varepsilon_{3}+p_{3}^{-1} K(\delta) \hat{z}_{2} .
$$

with $k_{3}>0$ and where

$$
\dot{\varepsilon}_{3}=z_{4}-\dot{\alpha}_{1}=\varepsilon_{4}-k_{3} \varepsilon_{3}-p_{3}^{-1} K(\delta) \hat{z}_{2} \stackrel{\text { def }}{=} \bar{f}_{3}(\mathbf{z}, \varepsilon, \hat{\mathbf{z}}) .
$$

With $V_{3}$ as in Eq. (28) we obtain

$$
\begin{aligned}
\dot{V}_{3}= & -W\left(z_{2}, \varepsilon_{3}\right)-p_{3} k_{3} \varepsilon_{3}^{2}+W_{z \tilde{z}}(\mathbf{z}, \tilde{\mathbf{z}})+\varepsilon_{3} K(\boldsymbol{\delta}) \tilde{z}_{2} \\
& +p_{3} \varepsilon_{3} \varepsilon_{4}+p_{4} \varepsilon_{4}\left(u+\alpha_{2}\right)
\end{aligned}
$$

where

$$
\alpha_{2}=k_{3}\left(z_{4}-\dot{\alpha}_{1}\right)-\ddot{\alpha}_{1}+p_{3}^{-1} \frac{d}{d t}\left[K(\delta) \hat{z}_{2}\right] .
$$

Using the control signal

$$
u=-k_{4} \varepsilon_{4}-\hat{\alpha}_{2}, \quad k_{4}>0
$$

we can rewrite Eq. (46) on the form

$$
\begin{aligned}
\dot{V}_{3} & =-W\left(z_{2}, \varepsilon_{3}\right)-p_{3} k_{3} \varepsilon_{3}^{2}-p_{4} k_{4} \varepsilon_{4}^{2}+p_{3} \varepsilon_{3} \varepsilon_{4}+ \\
& +W_{z \tilde{z}}(\mathbf{z}, \tilde{\mathbf{z}})+\varepsilon_{3} K(\delta) \tilde{z}_{2}+p_{4} \varepsilon_{4}\left(\alpha_{2}-\hat{\alpha}_{2}\right)
\end{aligned}
$$

where the term $\hat{\alpha}_{2}$ should approximate $\alpha_{2}$. We choose

$$
\hat{\alpha}_{2}=k_{3}\left(z_{4}-\dot{\alpha}_{1}\right)-\hat{\tilde{\alpha}}_{1}+p_{3}^{-1}\left(K(\boldsymbol{\delta}) \bar{f}_{2}+K^{\prime}(\delta)\left(z_{4}-\hat{z}_{2}\right) \hat{z}_{2}\right)
$$

where

$$
\begin{aligned}
& \hat{\hat{\alpha}}_{1}=\bar{f}_{2}+h^{\prime \prime}\left(\hat{z}_{2}\right) \bar{f}_{2}^{2}+h^{\prime}\left(\hat{z}_{2}\right)\left(-k \bar{f}_{1}-d \bar{f}_{2}+\right. \\
& \left.\left(K(\delta)+K^{\prime}(\delta)\left(\varepsilon_{3}+h\left(\hat{z}_{2}\right)\right)\right)\left(\bar{f}_{3}+h^{\prime}\left(\hat{z}_{2}\right) \hat{z}_{2}\right)\right) .
\end{aligned}
$$

With this choice and using (44), (47), (48) and (32)-(35), the error term $\left(\alpha_{2}-\hat{\alpha}_{2}\right)$ in Eq. (46) can be written

$\alpha_{2}-\hat{\alpha}_{2}=-\left(l_{1}+h^{\prime}\left(\hat{z}_{2}\right) l_{2}\right)\left(\tilde{z}_{2}-l_{1} \tilde{z}_{1}\right)-p_{3}^{-1} K(\delta) \tilde{z}_{2}\left(z_{2}-\tilde{z}_{2}\right)$.

Furthermore, since the observer error in Eq. (34)-(35) is exponentially stable, we can use the Lyapunov function

$$
V_{4}\left(z_{1}, z_{2}, \varepsilon_{3}, \varepsilon_{4}, \tilde{\mathbf{z}}\right)=V_{3}+\tilde{\mathbf{z}}^{T} \mathbf{P}_{\tilde{z}} \tilde{\mathbf{z}}, \quad \tilde{\mathbf{z}}=\left[\begin{array}{ll}
\tilde{z}_{1} & \tilde{z}_{2}
\end{array}\right]^{T}
$$

which gives

$$
\begin{aligned}
\dot{V}_{4}= & -W\left(z_{2}, \varepsilon_{3}\right)-p_{3} k_{3} \varepsilon_{3}^{2}-p_{4} k_{4} \varepsilon_{4}^{2}-\tilde{\mathbf{z}}^{T} \mathbf{Q}_{\tilde{z}} \tilde{\mathbf{z}}+p_{3} \varepsilon_{3} \varepsilon_{4} \\
& +W_{z \tilde{z}}(\mathbf{z}, \tilde{\mathbf{z}})+\varepsilon_{3} K(\boldsymbol{\delta}) \tilde{z}_{2}+p_{4} \varepsilon_{4}\left(\alpha_{2}-\hat{\alpha}_{2}\right)
\end{aligned}
$$

where $\mathbf{Q}_{\tilde{z}}>0$ is chosen freely and $\mathbf{P}_{\tilde{z}}>0$ satisfies the Lyapunov equation

$$
\mathbf{A}_{\tilde{z}}^{T} \mathbf{P}_{\tilde{z}}+\mathbf{P}_{\tilde{z}} \mathbf{A}_{\tilde{z}}=-\mathbf{Q}_{\tilde{z}}
$$

where $\mathbf{A}_{\tilde{z}}$ is defined by expressing (34)-(35) as $\dot{\tilde{\mathbf{z}}}=\mathbf{A}_{\tilde{z}} \tilde{\mathbf{z}}$.

The negative semi-definiteness of $\dot{V}_{4}$ in Eq. (51) could be established as in the state feedback case in Section IIIA, except for the presence of the last three extra crossterms in Eq. (51). Using the properties of $K$ and $h(x)$, and that the exponential convergence of the observer error gives that

$$
\left\|\tilde{z}_{2}(t)\right\| \leq \bar{v}, \quad \forall t \geq 0
$$

for some $\bar{v}>0$, the cross-terms can be bounded by

$$
\begin{aligned}
\left\|W_{z \tilde{z}}(\mathbf{z}, \tilde{\mathbf{z}})\right\| & \leq\left\|z_{2} \bar{K} \bar{h} \tilde{z}_{2}\right\|+\left\|z_{2} \bar{K} \tilde{z}_{1}\right\| \\
\left\|\varepsilon_{3} K(\boldsymbol{\delta}) \tilde{z}_{2}\right\| & \leq\left\|\varepsilon_{3} \bar{K} \tilde{z}_{2}\right\| \\
\left\|p_{4} \varepsilon_{4}\left(\alpha_{2}-\hat{\alpha}_{2}\right)\right\| & \leq\left\|\varepsilon_{4} p_{4}\left(\bar{h}_{l}+p_{3}^{-1} \bar{K} \bar{v}\right) \tilde{z}_{2}\right\|+ \\
& +\left\|\varepsilon_{4} p_{4} \bar{h}_{l} l_{1} \tilde{z}_{1}\right\|+\left\|\varepsilon_{4} p_{4} p_{3}^{-1} \bar{K} \bar{v} z_{2}\right\|
\end{aligned}
$$

with $\bar{h}_{l}=l_{1}+\bar{h} l_{2}$. By choosing $\mathbf{Q}_{\tilde{z}}$ sufficiently large, the cross-terms containing $\tilde{z}_{1}$ and $\tilde{z}_{2}$ can be dominated by the diagonal terms in $\dot{V}_{4}$. Straightforward calculations then show that if $p_{3} k_{3} k_{4}^{2} \geq(\bar{K} \bar{v})^{2} /(4 d)$ we can make $\dot{V}_{4}$ negative semi-definite by choosing $p_{4}=2 k_{4} p_{3}^{2} d /(\bar{K} \bar{v})^{2}$. Asymptotic stability of the origin follows from LaSalle's theorem [9], as in Section III-A.

\section{IMPLEMENTATION}

\section{A. Vision based observer}

In practice, the observer in Eqs. (32)-(33) should be able to compensate for positioning errors in all degrees of freedom. For this purpose, a dynamic model is obtained by extending the system in Eqs. (2)-(3) with a number of static states $\mathbf{x}_{s}$ with $\dot{\mathbf{x}}_{s}=0$, and interpret $z_{1}$ in Eq. (32) as the deviation from the nominal position $\mathbf{x}_{s}$. The extended model with $\mathbf{x}=\left(\mathbf{x}_{s}, z_{1}, z_{2}\right)^{T}$ is then used in an observer

$$
\dot{\hat{\mathbf{x}}}=\mathbf{F} \hat{\mathbf{x}}+\mathbf{G} \mathbf{f}_{c}+\mathbf{K}_{o}(t) \mathbf{J}_{p}^{\dagger}\left(\mathbf{y}-\mathbf{h}_{p}\left(\mathbf{x}_{s}, z_{1}\right)\right)
$$

where $\mathbf{f}_{c}$ is the vector of measured contact forces, and $\mathbf{J}_{p}^{\dagger}$ is the pseudo inverse of the Jacobian of the projection equation $\mathbf{h}_{p}$ for a standard pinhole camera. In the case of edge measurements, only the distances between the predicted and real edges in the normal direction of the contour are measurable, and the corresponding equations are obtained by projecting the image space errors onto the normal as in [10], [11]. Edge positions are found using a robust sub-pixel algorithm which localizes features at three different scales in the image, where visible features are predicted from frame to frame using a Binary Search Partitioning tree description of the object. The observer gain $\mathbf{K}_{o}(t)$ is obtained by using a time-varying Kalman filter. A multi-rate discrete-time approximation of the Kalman filter is used, where the dynamics and input forces are sampled at a significantly faster rate than the camera. It is assumed that the errors in the image measurements $\varepsilon_{y}$ can be modeled as Gaussian, spatially uncorrelated white noise with variance $\sigma^{2}$, so that an effective measurement error covariance $\varepsilon_{x}=\mathbf{J}_{p}^{\dagger} \varepsilon_{y}$ can be obtained from

$$
\mathrm{E}\left[\varepsilon_{x} \varepsilon_{x}^{T}\right]=\mathrm{E}\left[\mathbf{J}_{p}^{\dagger} \varepsilon_{y}\left(\mathbf{J}_{p}^{\dagger} \varepsilon_{y}\right)^{T}\right]=\left(\mathbf{J}_{p}^{T} \mathbf{J}_{p}\right)^{-1} \sigma^{2}
$$




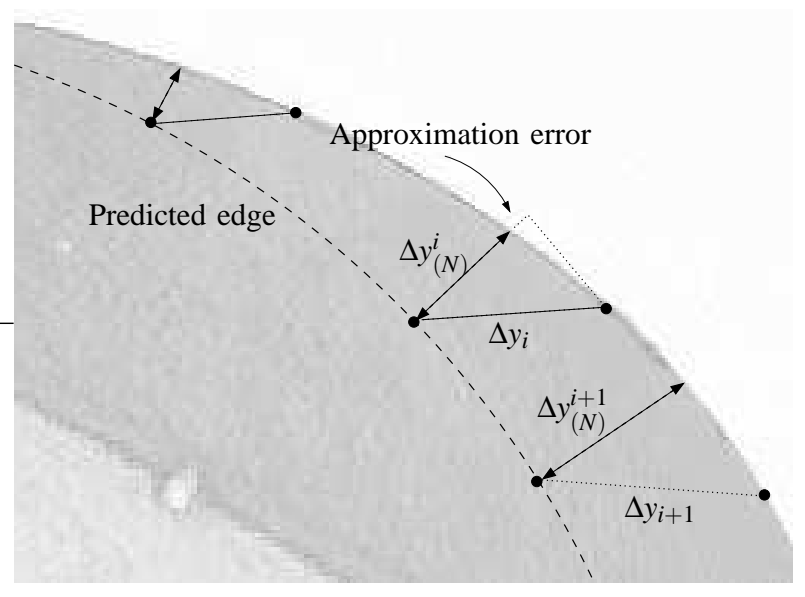

Fig. 2. Edge detection in the normal direction of the predicted edges.

\section{B. Control law}

The controller in Eq. (45) will damp the system by controlling the relative position of the robot tip and the contact point, which makes sense during the approach phase. Once contact has been established, it is necessary to switch to direct control of the contact force. The control law in Eq. (45) is given by

$$
\begin{aligned}
u= & -k_{4} \varepsilon_{4}-k_{3}\left(z_{4}-\dot{\alpha}_{1}\right)+\hat{\alpha}_{1}-p_{3}^{-1} \frac{d}{d t}\left[K(\delta) \hat{z}_{2}\right] \\
= & -\left(k_{3}+k_{4}\right)\left(z_{4}-\dot{\alpha}_{1}\right)-k_{3} k_{4}\left(z_{3}-\alpha_{1}\right) \\
& +\hat{\tilde{\alpha}}_{1}-k_{4} p_{3}^{-1} K(\delta) \hat{z}_{2}-p_{3}^{-1} \frac{d}{d t}\left[K(\delta) \hat{z}_{2}\right]
\end{aligned}
$$

where the first and second terms on the right hand side of (55) consist of feedback from the velocity- and position errors, respectively, while the third term can be seen as a compensation of the estimated environment acceleration. We now replace the position error $\left(z_{3}-\alpha_{1}\right)$ in (55) with the control error $e_{p}$ defined as

$$
e_{p}=\theta\left(\Psi^{-1}\left(f_{c}\right)\right) \Psi^{-1}\left(f_{c}\right)+\theta\left(\hat{z}_{1}-z_{3}\right) \cdot\left(z_{3}-\hat{z}_{1}\right)-h\left(\hat{z}_{2}\right)
$$

where $\theta(x)$ is the step function $\theta(x)=1, x \geq 0, \theta(x)=$ $0, x<0$. This leads to a switched control law approximating Eq. (45) which incorporates a proportional feedback from the measured force during the contact phase.

\section{RESULTS}

Simulations were carried out on a model of a three-link robot with a single point contact with the environment, and with contact forces controlled in the vertical direction only. The environment dynamics was given by a poorly damped mass-spring-damper system with mass $10 \mathrm{~kg}$, stiffness $2000 \mathrm{~N} / \mathrm{m}$, and linear damping of $3 \mathrm{~N} /(\mathrm{m} / \mathrm{s})$. The real contact stiffness $K(\boldsymbol{\delta})$ was set to $10000 \mathrm{~N} / \mathrm{m}$ for $\delta>0$. The damping function $h\left(\hat{z}_{2}\right)$ was composed of piecewise second

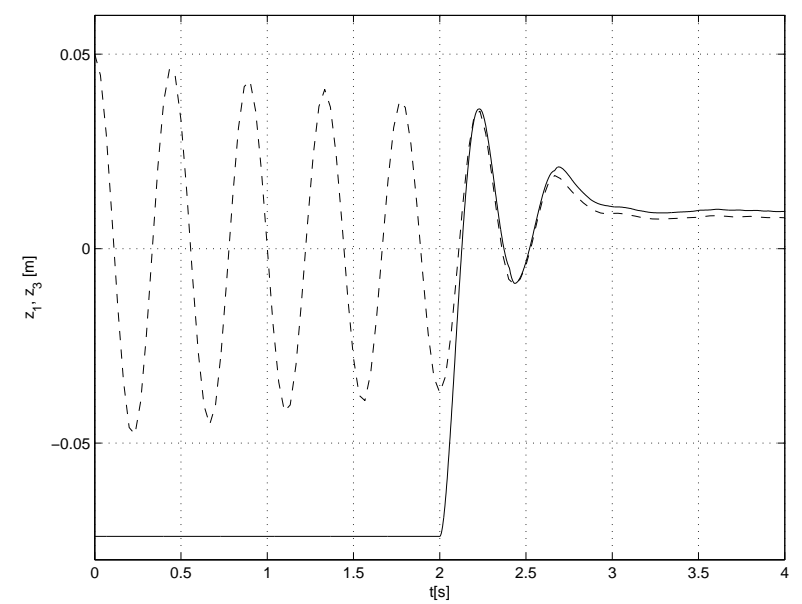

Fig. 3. Environment position $z_{1}$ (dashed) and robot tool tip position $z_{3}$ (solid) during simulated contact transition with active damping.

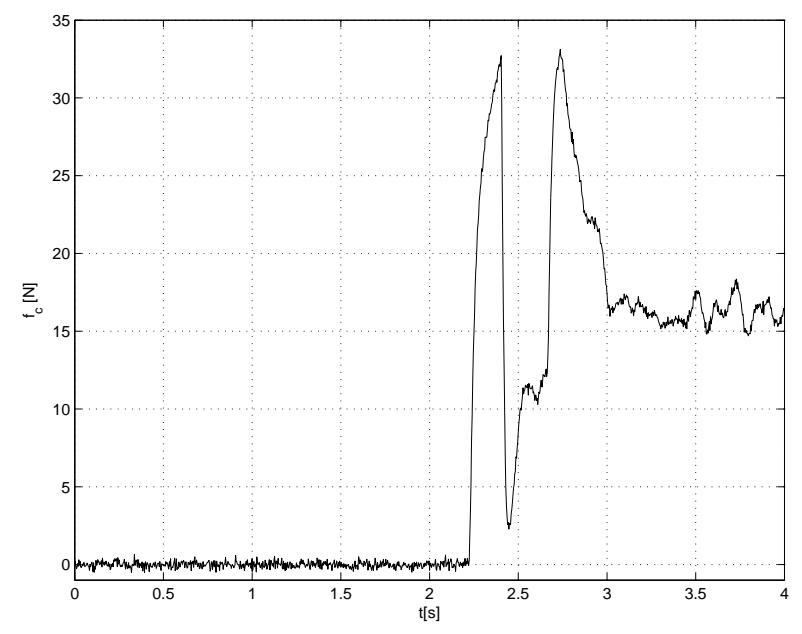

Fig. 4. Contact force $f_{c}$ during simulated contact transition and active damping.

order polynomials and chosen to correspond roughly to an additional damping of $d_{\text {active }}=200 \mathrm{~N} /(\mathrm{m} / \mathrm{s})$ in order to obtain a critically damped response for small velocities, while $h\left(\hat{z}_{2}\right)$ saturates at high velocities for a maximum desired contact force of $35 \mathrm{~N}$. An additional constant term was also added to the control signal in order to obtain a contact force $f_{c}=15 \mathrm{~N}$ in stationarity. The controller parameters were set to $k_{3}=k_{4}=20, p_{3}=40000$. The Kalman filter was set to track the translation of the workpiece, a textured cubic box with side $40 \mathrm{~cm}$, from synthetic $640 \times 480$ pixels camera images rendered in real time using the standard 3D graphics API OpenGL, see Fig. 5 for an example image.

In order to analyze the robustness of the system against modeling errors, a number of extra error sources were added in the simulation: 


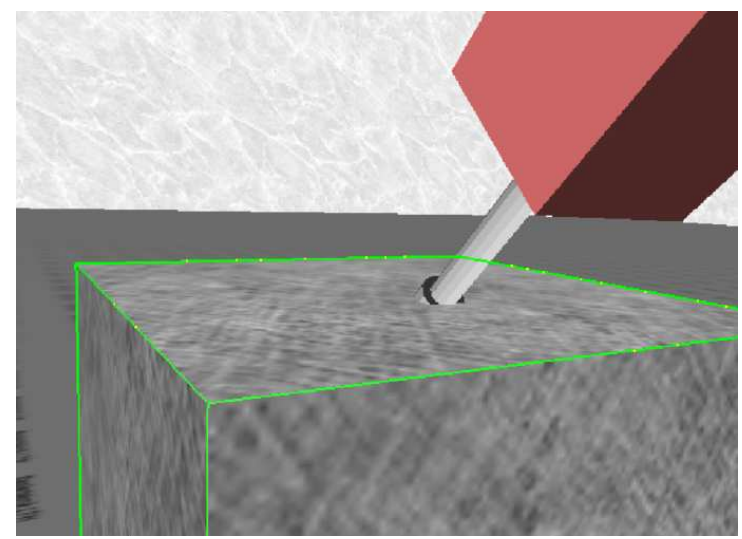

Fig. 5. Example of a rendered image, with superimposed wireframe model showing the estimated position of the cubic box.

- The model of $K(\delta)$ is a smoothed version of the true stiffness function, and the modeled elastic stiffness was $100 \%$ higher than the true value.

- Additional spatial noise was added to the synthetic images before the feature extraction step.

- The estimated value of parameter $d$ in the controller was $100 \%$ higher than the true value, while the stiffness $k$ was assumed to be known.

- The camera data and the observer were sampled at 40 $\mathrm{ms}$, while the force signal sampling and the damping control law were executed at a shorter period of $4 \mathrm{~ms}$. A time delay of $40 \mathrm{~ms}$ for image capture and processing was added for the camera data, and modeled in the Kalman filter.

For the motion control in the unconstrained directions a standard computed torque controller was used, with feedforward from the estimated environment position. The environment oscillation mode was excited, and at time $t=2 \mathrm{~s}$ the damping controller was started. Fig. 3 shows the resulting robot- and environment positions, while Fig. 4 shows the resulting contact force. The limiting of the contact force just below the desired value of $35 \mathrm{~N}$ can be seen at $t=2.4 \mathrm{~s}$, which is the reason why the desired critically damped dynamics is not achieved initially.

\section{Discussion}

The method works well in simulation, including some robustness to uncertainties in the model parameters $k, d$ and $K(\delta)$. The resulting controller has a relatively simple structure, as seen from Eqs. (55) and (56). Different choices of the damping function $h(x)$ will give different properties of the system. In practice, it is often possible and beneficial to choose a damping function that violates property (15), especially in order to obtain a sufficient damping for oscillations with small amplitude.

The role of the force sensor is twofold. Firstly, it is used to stiffen the controlled robot by force feed- forward, secondly to measure the current contact force for feedback. In order to perform force feed-forward, the assumption of a rigid manipulator restricts the method to environments which are significantly more compliant than the manipulator itself, or where the effective inertia of the robot is so large that the effect of interaction force can be ignored. Additionally, for very high contact stiffness between the robot and environment large contact forces can build up quickly, especially during the transition phase, meaning that additional physical compliance or padding may be necessary in order to decrease the contact stiffness.

\section{CONCLUSIONS}

In this paper, we have presented a method for vision/force-feedback control of a system consisting of a rigid manipulator interacting with an environment with linear dynamics. An observer-based backstepping control approach was used to find a controller that injects a desired damping into the dynamics of the environment, using both visual feedback and force sensing. Simulations were used to validate the approach. The choice of the controller parameters and damping function gives a considerable design flexibility, which can for instance be used to design damping controllers that attempt to limit the applied interaction force.

\section{REFERENCES}

[1] B. Siciliano and L. Villani, Robot Force Control, Kluwer Academic Publishers, 1999.

[2] N. Dioiaiti, C. Melchiorri, and S. Stramigioli, "Contact impedance estimation for robotic systems," in Proc. IEEE/RSJ Int. Conf. Intelligent Robots and Systems, Sendai, Japan, 2004, vol. 3, pp. 2538-2543.

[3] A. de Luca and C. Manes, "Modeling of robots in contact with a dynamic environment," IEEE Trans. Robotics and Automation, vol. 10, no. 4, pp. 542-548, August 1994.

[4] M. Vukobratovic, V. Potkonjak, and A. Rodic, "Contribution to the dynamic study of humanoid robots interacting with dynamic environment," Robotica, vol. 22, no. 4, pp. 439-447, 2004.

[5] J. Baeten, H. Bruyninckx, and J. De Schutter, "Combining eye-inhand visual servoing and force control in robotic tasks using the task frame," in Proc. IEEE Int. Conf. Multisensor Fusion, Taipei, Taiwan, August 1999, pp. 141-146.

[6] S. Jörg, J. Langwald, J. Stelter, G. Hirzinger, and C. Natale, "Flexible robot-assembly using a multi-sensory approach," in IEEE Int. Conf. Robotics and Automation, San Francisco, CA, USA, 2000, pp. 3687-3694.

[7] G. Morel, E. Malis, and S. Boudet, "Impedance based combination of visual and force control," in Proc. IEEE Int. Conf. Robotics and Automation, Leuven, Belgium, May 1998, pp. 1743-1748.

[8] J. Park and K Khatib, "Multi-link multi-contact force control for manipulators," in Proc. IEEE Int. Conf. Robotics and Automation, Barcelona, Spain, April 2005, pp. 3624-3629.

[9] M. Krstić, I. Kanellakopoulos, and P. V. Kokotović, Nonlinear and Adaptive Control Design, Wiley, New York, NY, 1995.

[10] T. Drummond and R. Cipolla, "Real-time tracking of complex structures with on-line camera calibration," in British Machine Vision Conf., 1999, vol. 2, pp. 574-583.

[11] F. Martin and R. Horaud, "Multiple camera tracking of rigid objects," Int. J. of Robotics Research, vol. 21, no. 2, pp. 97-113, February 2002. 